\title{
On Polyhedral Projection and Parametric Programming
}

\section{Journal Article}

\section{Author(s):}

Jones, C. N.; Kerrigan, E. C.; Maciejowski, J. M.

Publication date:

2008

Permanent link:

https://doi.org/10.3929/ethz-b-000014099

Rights / license:

In Copyright - Non-Commercial Use Permitted

Originally published in:

Journal of Optimization Theory and Applications 138(2), https://doi.org/10.1007/s10957-008-9384-4 


\title{
On Polyhedral Projection and Parametric Programming
}

\author{
C.N. Jones • E.C. Kerrigan · J.M. Maciejowski
}

Published online: 11 April 2008

(C) Springer Science+Business Media, LLC 2008

\begin{abstract}
This paper brings together two fundamental topics: polyhedral projection and parametric linear programming. First, it is shown that, given a parametric linear program (PLP), a polyhedron exists whose projection provides the solution to the PLP. Second, the converse is tackled and it is shown how to formulate a PLP whose solution is the projection of an appropriately defined polyhedron described as the intersection of a finite number of halfspaces. The input to one operation can be converted to an input of the other operation and the resulting output can be converted back to the desired form in polynomial time- this implies that algorithms for computing projections or methods for solving parametric linear programs can be applied to either problem class.
\end{abstract}

Keywords Parametric programming · Polyhedral projection · Computational geometry

Communicated by D.Q. Mayne.

E.C. Kerrigan's research was supported in part by the Royal Academy of Engineering, UK.

C.N. Jones $(\bowtie)$

Automatic Control Laboratory, ETH Zurich, Physikstrasse 3, Zurich, Switzerland

e-mail: cjones@ee.ethz.ch

E.C. Kerrigan

Department of Aeronautics and Department of Electrical and Electronic Engineering, Imperial

College London, Exhibition Road, London SW7 2AZ, UK

J.M. Maciejowski

Department of Engineering, University of Cambridge, Trumpington Street, Cambridge CB2 1PZ, UK 


\section{Introduction}

In this paper, we discuss the close relationship between the projection of polyhedra described as the intersection of a finite number of halfspaces and parametric linear programming. It is shown that an algorithm for one can be used to compute the solution to the other, and vice versa.

The calculation of the orthogonal projection of a polyhedron is a fundamental operation that arises in many applications. For example, in control theory, projection is required for reachability analysis [1] and in decision theory for the elimination of existential quantifiers [2]. It can be shown that the calculation of affine maps or Minkowski sums of polyhedra are both polynomially equivalent to orthogonal projection [3, Sect. 7.2], making a projection algorithm a useful and basic tool for working with polyhedra.

In recent years, there has been a surge of interest in the control community in parametric programming with vector-valued parameters. This interest has been motivated from the model predictive control (MPC) literature, since it can be shown that certain constrained, finite-horizon optimal control problems can be posed as parametric linear programs (PLPs) with the measured state as the parameter [4,5]. The use of PLPs allows the control action to be pre-computed off-line for every possible value of the parameter, which can drastically speed up the on-line implementation.

Beside the theoretical interest of linking these two operations, the importance of the work presented in this paper is clear: advances in projection algorithms are improvements in PLP algorithms and vice versa. Many problems have a specific structure that results in some algorithms being significantly faster than others. As a result of the material given here, a much larger range of both projection and PLP methods can be searched to find an approach that is efficient for a given problem.

There are currently four classes of algorithms for solving PLPs. The original in [6] enumerates all optimal bases of the problem using a method derived from the simplex algorithm. A similar algorithm was proposed in [7] in which all of the bases of the dual-constraints are enumerated. A geometric approach has been proposed $[4,5]$ that directly explores the set of admissible parameters in a recursive manner, subdividing the admissible parameter space into so-called critical regions, in which the set of active constraints at the optimizer does not change. This method can introduce a large number of artificial cuts in the parameter space and an extension of this algorithm in [8-10] partially addresses this problem. Finally, a new approach has been proposed in [11] that combines the efficient pivoting methods of [6] with the geometrically inspired algorithms of [8-10], resulting in a significantly faster approach.

Current projection methods that can operate in general dimensions can be grouped into four classes: Fourier elimination, block elimination, vertex enumeration and gift-wrapping approaches. Fourier-Motzkin elimination was originally described by Fourier in 1824 and can be thought of as the analogue of Gaussian elimination for linear inequalities. Several versions and improvements to Fourier's method have been proposed $([12,13]$ to name a few), although the primary contribution was due to Černikov [14] in 1963. In block elimination a polyhedron called the projection cone is defined whose extreme rays can then be used to find the defining halfspaces of the projection [15]. While there exist efficient methods for computing these extreme 
rays, (e.g. [16-18]), this approach may generate a large, and possibly exponential, number of redundant inequalities. It is also possible to enumerate the vertices of the polytope, compute their projection and then calculate the convex hull of the result. This approach can be efficient if there is a very small number of vertices, although it is possible that there may be an exponential number of vertices. The final approach enumerates the facets of the projection directly using a gift-wrapping approach [19, 20] and has been shown to be very efficient for a large class of polyhedra.

While a great deal has been published on projection and (vector-valued) parametric linear programming individually, to the best of the authors' knowledge, nothing has been published directly relating these two topics before. However, in a private communication with D. Klatte ${ }^{1}$ it was suggested that Fourier elimination was used to solve rudimentary PLPs in the 1970s, although no literature could be found on this topic. The interpretation of one dimensional parametric programming as projection appears in several texts $[21,22]$, although the multi-dimensional case has not been covered in the literature.

The remaining sections of this paper are organized as follows. Section 2 demonstrates the computation of parametric linear programs using a projection algorithm. Section 3 deals with the converse: a PLP is formulated whose output provides a desired projection. An example is given in Sect. 4 and final conclusions can be found in Sect. 5.

\subsection{Definitions and Notation}

A polyhedron is the intersection of a finite number of closed halfspaces,

$$
P \triangleq\left\{x \in \mathbb{R}^{n} \mid A x \leq b\right\}
$$

A polytope is a bounded polyhedron. If $A \in \mathbb{R}^{m \times n}$ is a matrix and $E \subseteq\{1, \ldots, m\}$ is a set of row indices, then $A_{E}$ is the matrix formed by the rows of $A$ whose indices are in $E$. If $E=\{i\}$ is a singleton, then we will write $A_{i}$ for $A_{\{i\}}$. Let

$$
P \triangleq\{x \mid A x \leq b\}
$$

be a polyhedron where $b \in \mathbb{R}^{m}$ and $E=\{1, \ldots, m\}$. The inequality $A_{i} x \leq b_{i}$ is $r e$ dundant for some $i \in\{1, \ldots, m\}$ if $\left\{x \mid A_{E \backslash\{i\}} x \leq b_{E \backslash\{i\}}\right\}=P$ and irredundant otherwise. If the set of inequalities $A x \leq b$ that describe a polytope are irredundant, then we call this description irredundant. Given a polyhedron $P \subseteq \mathbb{X} \times \mathbb{Y}$, where $\mathbb{X}$ and $\mathbb{Y}$ are subspaces, the projection of $P$ onto $\mathbb{X}$ is defined as

$$
\pi_{\mathbb{X}} P=\{x \in \mathbb{X} \mid \exists y \in \mathbb{Y},(x, y) \in P\} .
$$

Let $g: U \rightarrow \mathbb{R}$, where $U \subseteq \mathbb{R}^{n}$. The epigraph of $g$ is

$$
\operatorname{epi}(g) \triangleq\left\{(u, w) \in \mathbb{R}^{n} \times \mathbb{R} \mid u \in U, g(u) \geq w\right\} .
$$

\footnotetext{
${ }^{1}$ Prof. Dr. D. Klatte, Institut für Operations Research, Universität Zürich, Moussonstrasse 15, 8044 Zürich, klatte@ior.unizh.ch.
} 


\section{Solving Parametric Linear Programs via Projection}

In this section, we aim to solve the following parametric linear program:

$$
\begin{aligned}
& V^{\star}(\theta)=\min _{u} b^{T} u \\
& \text { s.t. } \quad(\theta, u) \in P,
\end{aligned}
$$

where $b \in \mathbb{R}^{n}$ is a vector, $\theta \in \Theta$ is the parameter and the constraints are defined by the polyhedron $P \subseteq \Theta \times \mathbb{U}$, where $\mathbb{U}=\mathbb{R}^{n}$ and $\Theta=\mathbb{R}^{d}$. By 'solving' the PLP, we mean computing an expression for the value function $V^{\star}(\cdot)$ and of an optimizer $u^{\star}(\cdot)$ for each value of the parameter $\theta$ as well as a description of the admissible set $\pi_{\Theta} P$.

Throughout this section we will make the assumption that the admissible set $\pi_{\Theta} P$ is full-dimensional and that the PLP (1) has a finite optimizer for every admissible value of the parameter $\theta \in \Theta$.

The availability of an algorithm to compute projections of polyhedra is assumed, and this section will show how to use such an algorithm to solve PLP (1). Given the matrices $C$ and $D$ and the vector $b$ that define a polytope

$$
Q \triangleq\{(x, y) \in \mathbb{X} \times \mathbb{Y} \mid C x+D y \leq b\},
$$

the projection algorithm returns a matrix $G$ and a vector $g$ such that

$$
\pi_{\mathbb{X}} Q=\{x \in \mathbb{X} \mid G x \leq g\} .
$$

The value function $V^{\star}(\cdot)$ is known to be continuous, piecewise affine and defined over a union of $d$-dimensional polyhedral regions, which form a cover of the set of admissible parameters $\pi_{\Theta} P$ [4]. In each region, the value function $V^{\star}(\cdot)$ is an affine function of $\theta$, and the optimizer is a set-valued function $u^{\star}(\cdot): \Theta \rightarrow 2^{\mathbb{U}}$ [4]. Solving a PLP therefore comes down to enumerating all such regions, as well as computing an expression for $V^{\star}(\cdot)$ and $u^{\star}(\cdot)$ in each region. The following theorem demonstrates that a projection operation can be used to solve a given PLP.

Theorem 2.1 Let the matrix $G \in \mathbb{R}^{N_{r} \times d}$ and the vector $g \in \mathbb{R}^{N_{r}}$ define the following irredundant description of the polyhedron:

$$
\begin{aligned}
& \left\{(\theta, J) \in \Theta \times \mathbb{J} \mid \mathbf{1} J \geq G \theta+g, \theta \in \pi_{\Theta} P\right\} \\
& \quad=\pi_{\Theta \times \mathbb{J}}\left\{(\theta, J, u) \in \Theta \times \mathbb{J} \times \mathbb{U} \mid J \geq b^{T} u,(\theta, u) \in P\right\},
\end{aligned}
$$

where $\mathbf{1}=[1 \cdots 1]^{T}$ is the vector of all ones, the set $\mathbb{J}$ is the real line $\mathbb{R}$ and the vector $b$ and the polyhedron $P$ are as defined in PLP (1). The solution of PLP (1) is defined over the following polyhedral regions:

$$
R_{i}=\left\{\theta \in \Theta \mid \mathbf{1}\left(G_{i} \theta+g_{i}\right) \geq G \theta+g, \theta \in \pi_{\Theta} P\right\}, \quad \forall i \in\left\{1, \ldots, N_{r}\right\} .
$$

In the region $R_{i}$, the value function $V^{\star}(\cdot)$ and the optimizer $u^{\star}(\cdot)$ are

$$
\begin{aligned}
V^{\star}(\theta) & =G_{i} \theta+g_{i}, \quad \forall \theta \in R_{i}, \\
u^{\star}(\theta) & =\left\{u \mid b^{T} u=G_{i} \theta+g_{i}, \quad(\theta, u) \in P\right\}, \quad \forall \theta \in R_{i} .
\end{aligned}
$$




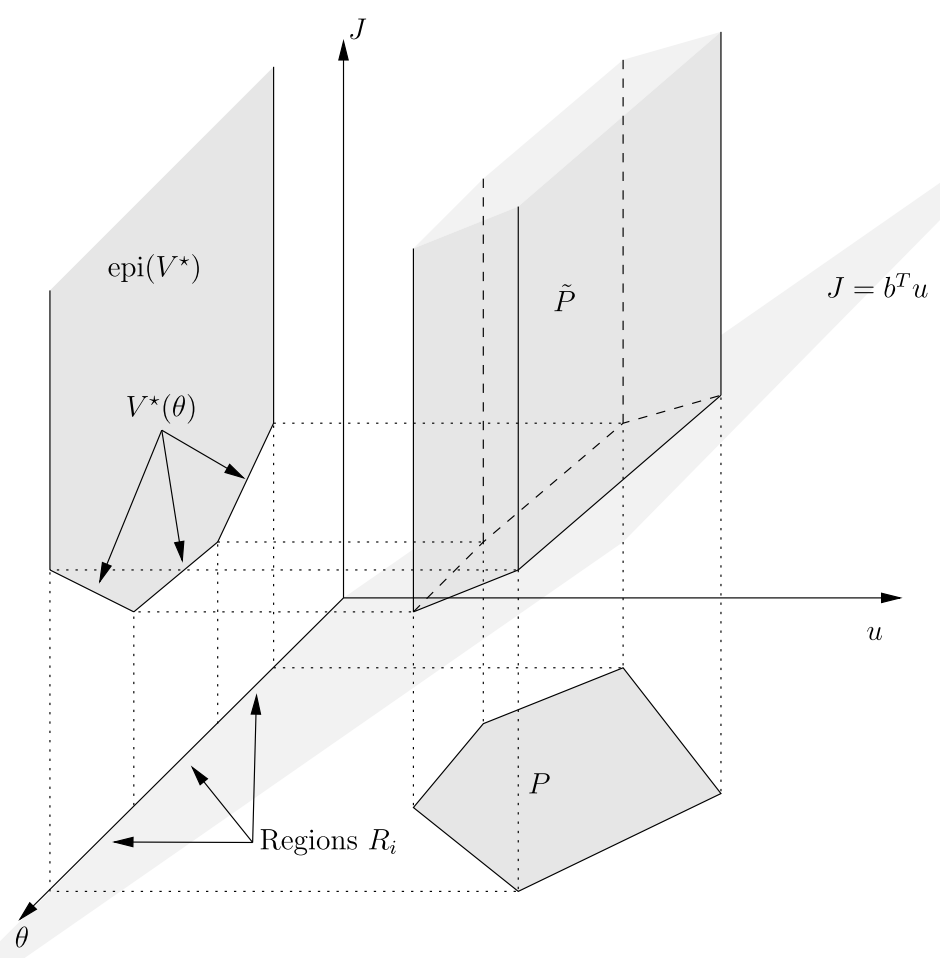

Fig. 1 Illustration of calculating a PLP using a projection operation

Proof We will proceed as in [23, Proposition 1.18] by constructing the epigraph of the function $V^{\star}(\cdot)$ using the projection operation. Second, we will show that the value function $V^{\star}(\cdot)$ and the polyhedral regions can be read directly from the epigraph and that the optimizer $u^{\star}(\cdot)$ can be readily computed from the expression for $V^{\star}(\cdot)$. The proposed procedure is illustrated in Fig. 1.

We begin by introducing an auxiliary variable $J \in \mathbb{J}$, where $\mathbb{J}=\mathbb{R}$, and rewriting the PLP (1) as

$$
V^{\star}(\theta)=\min _{u, J} J,
$$

s.t. $(\theta, J, u) \in \tilde{P}$,

where

$$
\tilde{P} \triangleq\left\{(\theta, J, u) \in \Theta \times \mathbb{J} \times \mathbb{U} \mid J \geq b^{T} u,(\theta, u) \in P\right\} .
$$


The dependence on the decision variable $u$ can now be removed through a projection operation,

$$
\begin{aligned}
& V^{\star}(\theta)=\min _{J} J, \\
& \text { s.t. } \quad(\theta, J) \in \pi_{\Theta \times \mathbb{J}} \tilde{P} .
\end{aligned}
$$

The polyhedron $\pi_{\Theta \times \mathbb{J}} \tilde{P}$ is clearly the epigraph of $V^{\star}(\cdot)$ and is unbounded above in the variable $J$, bounded below by $V^{\star}(\cdot)$ and bounded by the admissible region $\pi_{\Theta} P$ in the variable $\theta$. It follows that the epigraph can be written as

$$
\operatorname{epi}\left(V^{\star}\right)=\pi_{\Theta \times \mathbb{J}} \tilde{P}=\left\{(\theta, J) \in \Theta \times \mathbb{J} \mid \mathbf{1} J \geq G \theta+g, \theta \in \pi_{\Theta} P\right\}
$$

for some matrix $G \in \mathbb{R}^{N_{r} \times d}$ and vector $g \in \mathbb{R}^{N_{r}}$, which are returned by the projection algorithm.

The goal is now to use the epigraph epi $\left(V^{\star}\right)$ to compute the critical regions and the functions $V^{\star}(\cdot)$ and $u^{\star}(\cdot)$ for a given admissible parameter $\theta \in \pi_{\Theta} P$.

From (4)-(5), the value function is given by

$$
V^{\star}(\theta)=\max _{i \in\left\{1, \ldots, N_{r}\right\}} G_{i} \theta+g_{i}
$$

The value function is clearly piecewise affine and each of the $m$ polyhedral regions in which it is affine is given by

$$
\begin{aligned}
R_{i} & \triangleq \pi_{\Theta}\left(\left\{(\theta, J) \in \Theta \times \mathbb{J} \mid G_{i} \theta+g_{i}=J\right\} \cap \pi_{\Theta \times \mathbb{J}} \tilde{P}\right) \\
& =\left\{\theta \in \Theta \mid \mathbf{1}\left(G_{i} \theta+g_{i}\right) \geq G \theta+g, \theta \in \pi_{\Theta} P\right\}, \quad \forall i \in\left\{1, \ldots, N_{r}\right\} .
\end{aligned}
$$

By definition, the optimizer in the $i$ th region is then given by the points $u \in \mathbb{U}$ that achieve the value function

$$
\begin{aligned}
u^{\star}(\theta) & =\left\{u \in \mathbb{U} \mid b^{T} u=V^{\star}(\theta),(\theta, u) \in P\right\} \\
& =\left\{u \in \mathbb{U} \mid b^{T} u=G_{i} \theta+g_{i},(\theta, u) \in P\right\}, \quad \forall \theta \in R_{i}, i \in\left\{1, \ldots, N_{r}\right\}
\end{aligned}
$$

Remark 2.1 The assumption is not made that the projection algorithm returns an irredundant description of the projection. However, the regions resulting from redundant inequalities of the projection will be empty in Theorem 2.1 and so in general an irredundant description would be preferred.

Note that the optimizer $u^{\star}: \Theta \rightarrow 2^{\mathbb{U}}$ is in general a set-valued function. From (7) and (6), one can see that it is also continuous, since the value function $V^{\star}$ is singlevalued and continuous. Section 2.1 shows how to modify the problem formulation such that a single-valued, continuous selection is made from the set of optimizers. i.e. a function $\hat{u}^{\star}(\theta): \Theta \rightarrow \mathbb{U}$ is computed such that $\hat{u}^{\star}(\theta) \in u^{\star}(\theta)$ for all $\theta \in \Theta$. 
Remark 2.2 If the optimizer is single-valued in a given region, it is often desirable to write it as an affine function of $\theta$. This can easily be achieved by solving the PLP (1) once for each region for a fixed value of the parameter that has been selected from the interior of the given region. If the constraint polyhedron $P$ is defined as

$$
P=\{(\theta, u) \in \Theta \times \mathbb{U} \mid C u+D \theta \leq b\}
$$

and if the active constraints at the optimum are $E$, then the optimizer in the region $R_{i}$ is given by [24]

$$
u^{\star}(\theta)=C_{E}^{\dagger}\left(b_{E}-D_{E} \theta\right), \quad \forall \theta \in R_{i}
$$

where $^{\dagger}$ denotes the Moore-Penrose pseudoinverse.

\subsection{Degeneracy}

A region $R_{i}$ is called dual-degenerate if the optimizer $u^{\star}(\theta)$ is set-valued for some $\theta$ in the relative interior of $R_{i}$. Some applications require that there be a single, unique optimizer such as in many control problems, where the optimizer is the control input [5, 24]. Methods for obtaining a unique optimizer for PLPs were presented in $[11,25]$. The solution in [25] is similar to that proposed here in that the author solves in the degenerate region $R_{i}$, a parametric quadratic program with a positive definite cost, which guarantees a unique solution. In [11] the problem is symbolically perturbed such that the perturbed problem is no longer dual degenerate. It should be noted that both of these approaches guarantee that the selected optimizer will be continuous, which is a valuable property in many applications.

A unique solution can also be guaranteed through the recursive application of the procedure introduced in this paper, as detailed in the following. If $R_{i}$ is a dual degenerate region and $V^{\star}(\theta)=G_{i} \theta+g_{i}$ is the value function in the region $R_{i}$, then we solve the following parametric linear program over the set of optimizers in that region:

$$
\begin{array}{ll}
f(\theta)=\min _{u} \check{b}^{T} u, \\
\text { s.t. } & (\theta, u) \in P, \\
& b^{T} u=G_{i} \theta+g_{i}, \\
& \theta \in R_{i},
\end{array}
$$

where $\check{b}$ is any nonzero vector, different from $b$.

The parametric linear program (8) is defined only for $\theta \in R_{i}$ and the solution is clearly optimal for PLP (1). Further, if $\breve{b}$ is selected such that it is not perpendicular to any edge of $P$, then PLP (8) will not be dual-degenerate and the solution will be single-valued. This assumption can normally be met by choosing $\breve{b}$ randomly, or can be guaranteed by treating it as a symbolic perturbation vector [11]. 


\section{Solving Projection via Parametric Linear Programming}

In this section we further investigate the relationship between projection and parametric programming by developing a method of computing projections given any parametric linear programming algorithm that takes as input the data of PLP (1) and returns a piecewise-affine description of the value function $V^{\star}$. Specifically, given a polyhedron $P \subseteq \mathbb{X} \times \mathbb{Y}$, we aim to compute an irredundant description of the projection $\pi_{\mathbb{X}} P$ using an algorithm that can compute the solution to a PLP. In this section, we will define the polyhedron $P$ using the matrices $C \in \mathbb{R}^{n \times d}$ and $D \in \mathbb{R}^{n \times k}$ and the vector $b \in \mathbb{R}^{n}$ as

$$
P \triangleq\{(x, y) \in \mathbb{X} \times \mathbb{Y} \mid C x+D y \leq b\},
$$

where $\mathbb{X}=\mathbb{R}^{d}$ and $\mathbb{Y}=\mathbb{R}^{k}$.

We begin with the projection lemma, which is often attributed to Černikov [14] and can be derived directly from Farkas lemma (see for example [3]).

Lemma 3.1 (Projection Lemma) If $P=\{(x, y) \in \mathbb{X} \times \mathbb{Y} \mid C x+D y \leq b\}$ is a polyhedron, then the projection of $P$ onto $\mathbb{X}$ is

$$
\pi_{\mathbb{X}} P=\left\{x \in \mathbb{X} \mid w^{T} C x \leq w^{T} b, \forall w \in W\right\},
$$

where $W$ is called the projection cone and is defined as

$$
W \triangleq\left\{w \in \mathbb{R}^{n} \mid D^{T} w=0, w \geq 0\right\} .
$$

One standard approach to computing the projection is to first enumerate the extreme rays of the projection cone $W$ using any number of standard tools (for example $[16,17])$, write down the projection $\pi_{\mathbb{X}} P$ from (9), and then to remove redundancies. This approach is often inefficient because many of the extreme rays of $W$ can generate redundant inequalities of the projection and for many problems there is an exponential number of redundant inequalities generated. The procedure described here uses a PLP to enumerate only those rays of the projection cone that generate irredundant inequalities of $\pi_{\mathbb{X}} P$.

Lemma 3.2 If $S$ is the set

$$
S \triangleq\left\{(\alpha, \beta) \in \mathbb{R}^{d} \times \mathbb{R} \mid \exists w \in W, \quad\left[\begin{array}{l}
\alpha \\
\beta
\end{array}\right]=\left[\begin{array}{l}
C^{T} \\
b^{T}
\end{array}\right] w\right\}
$$

and if $P \triangleq\{(x, y) \in \mathbb{X} \times \mathbb{Y} \mid C x+D y \leq b\}$, then

$$
\left\{x \mid \alpha x \leq b,(\alpha, \beta) \in \operatorname{extr} S,\left\|\left[\alpha^{T} \beta\right]\right\|_{2}=1\right\}
$$

is an irredundant description of $\pi_{\mathbb{X}} P$, where extr $S$ is the set of extreme rays of $S$.

Proof From Lemma 3.1 we can see that for every $(\alpha, \beta) \in S$, the inequality $\alpha^{T} x \leq \beta$ is satisfied for all $x$ in the projection $\pi_{\mathbb{X}} P$, or in other words, it is valid. Furthermore, 
the set of coefficients of the irredundant inequalities of $\pi_{\mathbb{X}} P$ is a subset of $S$. We note that an inequality $\alpha^{T} x \leq \beta$ of $\pi_{\mathbb{X}} P$ is irredundant if it cannot be written as the positive combination of valid inequalities [27]. Equivalently, $\left\{t\left[\alpha^{T} \beta\right]^{T} \mid t \geq 0\right\}$ is an extreme ray of $S$ if and only if it cannot be written as a positive combination of other elements of $S$. It follows that the extreme rays of $S$ define the irredundant halfspaces of $\pi_{\mathbb{X}} P$ up to scaling of the ray. Finally, the constraint

$$
\left\|\left[\begin{array}{ll}
\alpha^{T} & \beta
\end{array}\right]\right\|_{2}=1
$$

simply chooses a particular scaling.

The goal of computing all irredundant inequalities of $\pi_{\mathbb{X}} P$ is now reduced to finding all extreme rays of $S$. As will be seen shortly, the problem of finding all vertices of a polytope (bounded polyhedron) can be posed as a parametric linear program and therefore, if we wish to use a PLP, we must first bound the cone $S$. We assume a vector $a$ such that for every point $(\alpha, \beta) \neq(0,0)$ in $S$ we have $0<\left[\alpha^{T} \beta\right] a<\infty$. The existence of such a vector $a$ is proven by the following lemma.

Lemma 3.3 If $S$ is as defined in (10), then there exists a vector a such that $0<$ $\left[\alpha^{T} \beta\right] a<\infty$ for all $(\alpha, \beta) \neq(0,0)$ in $S$ if $a$ is in the set

$$
\left\{a \in \mathbb{R}^{d+1} \mid\|a\|_{2}<\infty, \exists y \in \mathbb{Y}, D y \leq\left[\begin{array}{ll}
C & b
\end{array}\right] a\right\} .
$$

Proof Let $a$ be in the set $\left\{a \in \mathbb{R}^{d+1} \mid \exists y \in \mathbb{Y},\|a\|_{2}<\infty\right.$, let $\left.D y \leq[C b] a\right\}$ and let $w$ be in the projection cone $W$. Since $w$ is positive, the inequality

$$
w D y \leq w[C \quad b] a
$$

holds for all $w$. We have that $w D=0$ for all $w$ in $W$ and recalling that

$$
\left[\begin{array}{ll}
\alpha^{T} & \beta
\end{array}\right]=w\left[\begin{array}{ll}
C & b
\end{array}\right]
$$

gives the desired result.

Remark 3.1 An appropriate vector $a^{\star}$ can be found by, for example, computing the Chebychev center of the cone from Lemma 3.3, while restricting the radius to one,

$$
\begin{aligned}
& \left(u^{\star}, a^{\star}, t^{\star}\right)=\underset{u, a, t}{\operatorname{argmax}} t, \\
& \text { s.t. } \quad D u-\left[\begin{array}{ll}
C & b
\end{array}\right] a+\left\|\left[\begin{array}{lll}
D & -C & -b
\end{array}\right]\right\|_{2}^{2} t \leq 0, \quad 0 \leq t \leq 1,
\end{aligned}
$$

where the 2-norm is taken row-wise and an appropriate $a$ exists if and only if $t>0$.

Remark 3.2 Note that the set $S$ is the homogenization of the polar dual of $\pi_{\mathbb{X}} P$. As such, if the polyhedron $P$ is bounded, then a suitable vector $a$ is given by

$$
a=\left[\begin{array}{llll}
0 & \ldots & 0 & 1
\end{array}\right]^{T} .
$$

See [3] for a discussion on homogenizations and polar duals. 
The set $S$ can now be bounded by the inclusion of the constraint $\left[\alpha^{T} \beta\right] a=1$,

$$
\bar{S} \triangleq\left\{(\alpha, \beta) \mid a^{T}\left[\begin{array}{l}
\alpha \\
\beta
\end{array}\right]=1\right\} \cap S .
$$

Since $S$ is a cone, each ray of $S$ intersects the hyperplane $\left[\alpha^{T} \beta\right] a=1$ exactly once, and it is clear that there is a one-to-one correspondence between vertices of $\bar{S}$ and rays of $S$.

Before stating the main result of this section, the following standard result is needed.

Theorem $3.1 x_{0} \in \mathbb{R}^{n}$ is an extreme point of a polytope $P$ if and only if, for some vector $c \in \mathbb{R}^{n}$, we have $\max \left\{c^{T} x \mid x \in P\right\}=c^{T} x_{0}>c^{T} x$ for all $x \in P, x \neq x_{0}$.

Proof See for example [28, Sect. 7.2(d)].

The following theorem will allow the irredundant inequalities of the projection to be computed using a parametric linear program.

Theorem 3.2 Let $P \triangleq\{(x, y) \in \mathbb{X} \times \mathbb{Y} \mid C x+D y \leq b\}$ be a polyhedron. Consider the following PLP:

$$
\begin{aligned}
& V^{\star}(\theta)=\max _{\alpha, \beta} \theta^{T}\left[\begin{array}{l}
\alpha \\
\beta
\end{array}\right], \\
& \text { s.t. } \quad(\alpha, \beta) \in \bar{S},
\end{aligned}
$$

where $\bar{S}$ is as defined in (12). If the piecewise linear function $V^{\star}(\cdot)$ is defined over $N_{r}$ polyhedral regions and the cost in the $i$ th region is given by $V^{\star}(\theta)=\left[F_{i} f_{i}\right] \theta$, then an irredundant description of the projection of $P$ onto $\mathbb{X}$ is given by

$$
\pi_{\mathbb{X}} P=\{x \in \mathbb{X} \mid F x \leq f\} .
$$

Proof From Theorem 3.1, it can be seen that given a polytope $Q$, if $\operatorname{argmax}\left\{c^{T} u \mid\right.$ $u \in Q\}$ is computed for every cost vector $c$, then all vertices of $Q$ will be enumerated. One can see therefore that parametric linear program (13) will enumerate the vertices of the set $\bar{S}$ from (12).

For every vertex $\left[\alpha_{\circ}^{T} \beta_{\circ}\right]^{T}$ of $\bar{S}$ there exists a full-dimensional polyhedral set $R$ such that for all $\theta$ in $R$, the vertex $\left[\alpha_{\circ}^{T} \beta_{\circ}\right]^{T}$ is the optimizer of PLP (13). As discussed in Sect. 2, the solution to a PLP is defined over precisely these regions. The value function for all $\theta$ in region $R$ is clearly

$$
V^{\star}(\theta)=\left[\begin{array}{ll}
\alpha_{\circ}^{T} & \beta_{\circ}
\end{array}\right] \theta=\left[\begin{array}{ll}
F_{i} & f_{i}
\end{array}\right] \theta
$$

and therefore the theorem follows directly from Lemma 3.1 and Theorem 3.1.

Remark 3.3 The PLP given in Theorem 3.2 has the parameter in the cost, rather than on the right hand side of the constraints as in Sect. 2, which is more common in the 
Fig. 2 Epigraph of PLP (15) and regions $R_{i}$

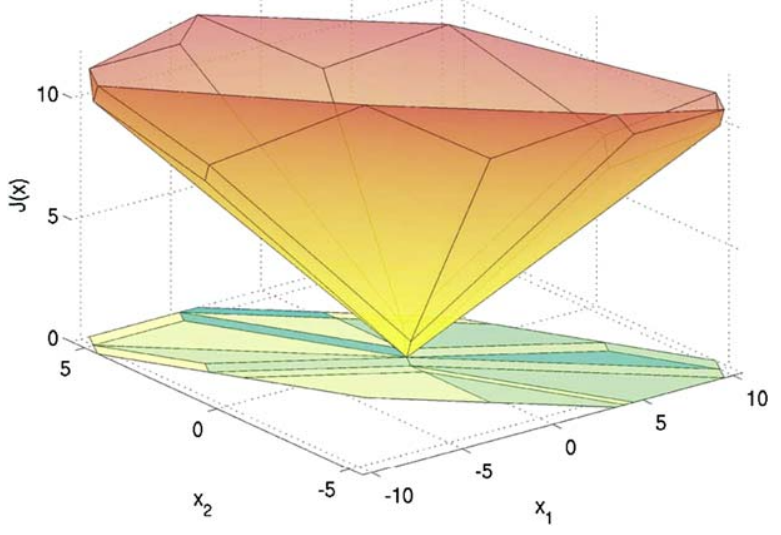

literature. The PLP (13) can be written in a more common form by posing its dual

$$
\begin{aligned}
& V^{\star}(\theta)=\min _{y, t} t, \\
& \text { s.t. } \quad D y+\left[\begin{array}{ll}
C & b
\end{array}\right] a t \leq\left[\begin{array}{ll}
C & b
\end{array}\right] \theta .
\end{aligned}
$$

Note also that the value function $V^{\star}$ in (13) is piecewise linear, rather than piecewise affine since the cost function $\theta^{T}\left[\alpha^{T} \beta\right]^{T}$ does not have an affine term.

\section{Example}

Consider the following PLP, which is motivated by the commonly used control example of the double-integrator [29]:

$$
\begin{aligned}
& V^{\star}(x)=\min _{u, s} \mathbf{1}^{T} s, \\
& \text { s.t. } \quad-\gamma \leq \Lambda x+\Sigma u \leq \gamma, \\
& \quad-\Psi_{s} \leq \Lambda x+\Sigma u \leq \Psi s, \\
& \quad s \geq 0,
\end{aligned}
$$

where $x \in \mathbb{X}=\mathbb{R}^{2}$ and we have defined the following matrices for simplicity:

$$
\begin{array}{llll}
\gamma \triangleq\left[\begin{array}{llllll}
10 & 10 & 10 & 10 & 2 & 2
\end{array}\right]^{T} & \Lambda \triangleq\left[\begin{array}{lllllll}
2 & 0 & 2 & 0 & 0 & 0 \\
2 & 2 & 4 & 2 & 0 & 0
\end{array}\right]^{T}, \\
\Sigma \triangleq\left[\begin{array}{lllllll}
2 & 1 & 3 & 1 & 2 & 0 \\
0 & 0 & 2 & 1 & 0 & 2
\end{array}\right]^{T} & \Psi \triangleq\left[\begin{array}{llllll}
1 & 1 & 0 & 0 & 0 & 0 \\
0 & 0 & 1 & 1 & 0 & 0 \\
0 & 0 & 0 & 0 & 1 & 1
\end{array}\right]^{T} .
\end{array}
$$


Fig. 3 Admissible region of PLP (15), computed using PLP (18)

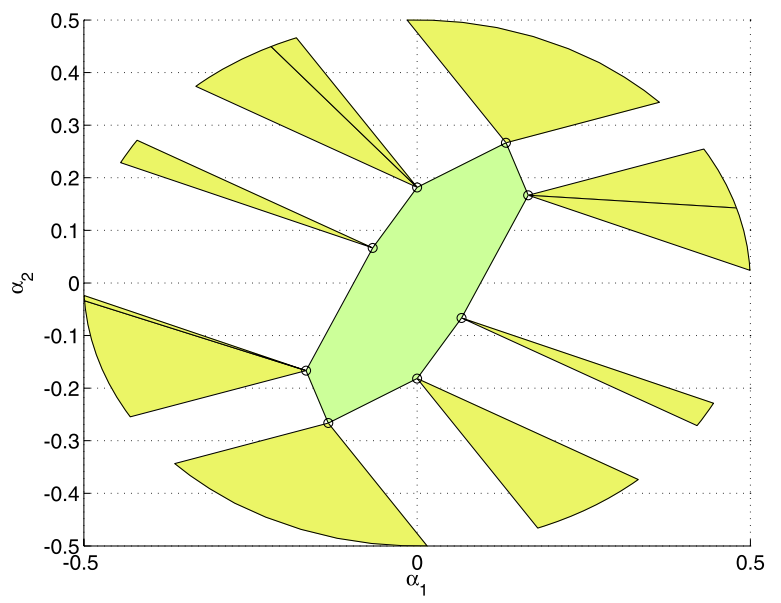

(a) Critical regions, which are also the normal fan of the polar dual of the projection

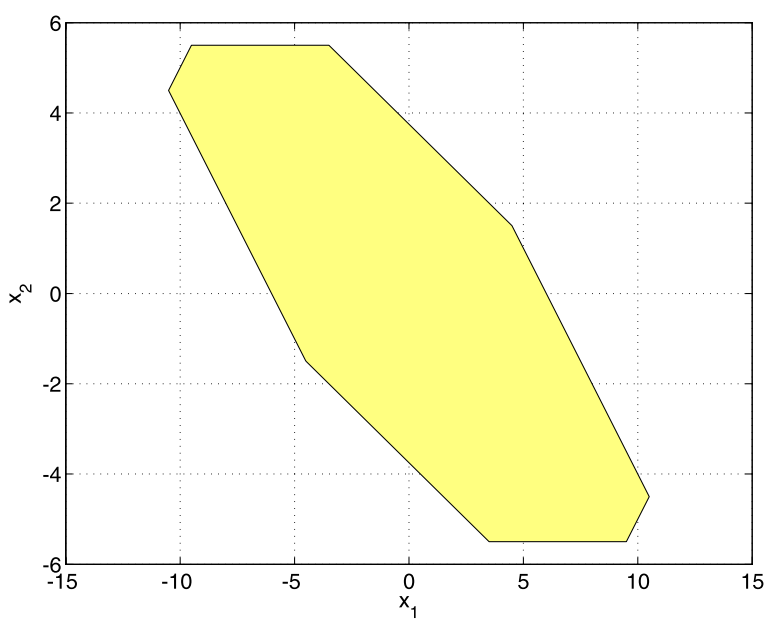

(b) Admissible region of PLP (15)

Equation (5) can now be used to formulate the PLP as a projection,

$$
\begin{aligned}
& \operatorname{epi}\left(V^{\star}\right)=\pi_{\mathbb{X} \times \mathbb{J}} \tilde{P} \\
& =\pi_{\mathbb{X} \times \mathbb{J}}\left\{(x, J, u, s) \mid \begin{array}{c}
-\gamma \leq \Lambda x+\Sigma u \leq \gamma \\
-\Psi s \leq \Lambda x+\Sigma u \leq \Psi_{s} \\
s \geq 0 \\
J \geq \mathbf{1}^{T} s
\end{array}\right\} .
\end{aligned}
$$

A plot of epi $\left(V^{\star}\right)$ is shown in Fig. 2, along with the polyhedral regions $R_{i}$ of the PLP (15). 
The admissible region of the PLP (15) is given by the projection

$$
\pi_{\mathbb{X}}\{(x, U) \mid-\gamma \leq \Lambda x+\Sigma U \leq \gamma\}
$$

This projection can be computed as in (13) through solving the following parametric linear program:

$$
\begin{aligned}
& V^{\star}(x)=\max _{\lambda} x^{T}\left[\Lambda^{T}-\Lambda^{T}\right] \lambda, \\
& \text { s.t. } \quad\left[\Sigma^{T}-\Sigma^{T}\right] \lambda=0, \\
& \quad \lambda \geq 0,
\end{aligned}
$$

where we have taken advantage of the admissible region being bounded to select $a=\left[\begin{array}{ll}0 & 1\end{array}\right]^{T}$. Figure 3 shows the regions of the solution, as well as the corresponding projection.

\section{Conclusions}

This note has shown how to use a parametric linear programming algorithm to compute polyhedral projections and vice versa. Since many projection and PLP algorithms are particularly suited to problems of a specific structure, this result will allow a much larger range of both projection and PLP algorithms to be examined to find one that matches the structure of a given problem. Furthermore, as both the input and output of the two problems can be transformed from one to the other in polynomial time, any efficient algorithm for solving PLPs is now an equally efficient algorithm for solving the projection problem and vice versa.

\section{References}

1. Blanchini, F.: Set invariance in control-a survey. Automatica 35(11), 1747-1768 (1999)

2. Vidal, R., Schaffert, S., Lygeros, J., Sastry, S.: Controlled invariance of discrete time systems. In: Hybrid Systems: Computation and Control. Lecture Notes in Computer Science, vol. 1790, pp. 437450. Springer, Berlin (2000)

3. Ziegler, G.M.: Lectures on Polytopes. Springer, New York (1995)

4. Borrelli, F., Bemporad, A., Morari, M.: A geometric algorithm for multi-parametric linear programming. J. Optim. Theory Appl. 118(3), 515-540 (2003)

5. Bemporad, A., Borrelli, F., Morari, M.: Model predictive control based on linear programming-the explicit solution. IEEE Trans. Autom. Control 47(12), 1974-1985 (2002)

6. Gal, T.: Postoptimal Analyses, Parametric Programming and Related Topics, 2nd edn. de Gruyter, Berlin (1995)

7. Schechter, M.: Polyhedral functions and multiparametric linear programming. J. Optim. Theory Appl. 53(2), 269-280 (1987)

8. Baotić, M.: An efficient algorithm for multi-parametric quadratic programming. Technical report, ETH Zürich, Institut für Automatik, Physikstrasse 3, CH-8092, Switzerland (2002)

9. Grieder, P., Borrelli, F., Torrisi, F., Morari, M.: Computation of the constrained infinite time linear quadratic regulator. Automatica 40, 701-708 (2004)

10. Tøndel, P., Johansen, T.A., Bemporad, A.: An algorithm for multi-parametric quadratic programming and explicit MPC solutions. In: Proceedings of the 40th IEEE Conference on Decision and Control, Orlando, FL, USA (2001) 
11. Jones, C.N., Kerrigan, E.C., Maciejowski, J.M.: Lexicographic perturbation for multiparametric linear programming with applications to control. Automatica 43(10), 1608-1816 (2007)

12. Jaffar, J., Maher, M.J., Stuckey, P.J., Yap, R.H.C.: Projecting $\operatorname{clp}(\mathcal{R})$ constraints. New Gener. Comput. 11(3,4), 449-469 (1993)

13. Ponce, J., Sullivan, S., Sudsang, A., Boissonnat, J., Merlet, J.: On computing four-finger equilibrium and force-closure grasps of polyhedral objects. Int. J. Robot. Res. 16(1), 11-35 (1997)

14. Černikov, S.N.: Contraction of finite systems of linear inequalities. Dokl. Akad. Nauk SSSR 152(5), 1075-1078 (1963) (in Russian). (English translation in Soc. Math. Dokl. 4(5), 1520-1524 (1963))

15. Balas, E., Pulleybank, W.R.: The perfectly matchable subgraph polytope of a bipartite graph. Networks 13, 495-516 (1983)

16. Fukuda, K., Prodon, A.: Double description method revisited. In: Deza, M., Euler, R., Manoussakis, I. (eds.) Combinatorics and Computer Science. Lecture Notes in Computer Science, vol. 1120, pp. 91-111. Springer, Berlin (1996). Postscript file available from ftp://ftp.ifor.math.ethz.ch/pub/ fukuda/reports/ddrev960315.ps.gz

17. Avis, D., Fukuda, K.: A pivoting algorithm for convex hulls and vertex enumeration of arrangements and polyhedra. Discrete Comput. Geom. 8, 295-313 (1992)

18. Avis, D., Fukuda, K.: Reverse search for enumeration. Discrete Appl. Math. 65, 21-46 (1996)

19. Amenta, N., Ziegler, G.M.: Shadows and slices of polytopes. In: Proceedings of the 12th Annual Symposium on Computational Geometry, pp. 10-19. ACM Press, New York (1996)

20. Jones, C.N., Kerrigan, E.C., Maciejowski, J.M.: Equality set projection: a new algorithm for the projection of polytopes in halfspace representation. Technical Report CUED/F-INFENG/TR. 463, Department of Engineering, University of Cambridge, 2004. http://www-control.eng.cam.ac.uk/ cnj22/docs/resp_mar_04_15.pdf

21. Klee, V., Kleinschmidt, P.: Geometry of the Gass-Saaty parametric cost LP algorithm. Discrete Comput. Geom. 5, 13-26 (1990)

22. Murty, K.G.: Linear Programming. Wiley, New York (1983)

23. Tyrrell Rockafellar, R., Wets, R.J.-B.: Variational Analysis. A series of Comprehensive Studies in Mathematics, vol. 317. Springer, Berlin (1998)

24. Borrelli, F.: Constrained Optimal Control Of Linear And Hybrid Systems. Lecture Notes in Control and Information Sciences, vol. 290. Springer, Berlin (2003)

25. Spjøtvold, J., Tøndel, P., Johansen, T.A.: A method for obtaining continuous solutions to multiparametric linear programs. In: Proceedings of the 16th IFAC World Congress, Prague (2005)

26. Jones, C.N.: Polyhedral Tools for Control. PhD thesis, University of Cambridge, July 2005

27. Cheng, M.C.: General criteria for redundant and nonredundant linear inequalities. J. Optim. Theory Appl. 53(1), 37-42 (1987)

28. Padberg, M.: Linear Optimization and Extensions. Algorithms and Combinatorics. Springer, Berlin (1999)

29. Bemporad, A., Borrelli, F., Morari, M.: Min-max control of constrained uncertain discrete-time linear systems. IEEE Trans. Autom. Control 48(9), 1600-1606 (2003) 